\title{
Trematódeos emergentes de moluscos dulciaquícolas coletados em valas, no município de Peruíbe, estado de São Paulo, Brasil
}

\author{
Trematodes emerging from freshwater mollusks collected in ditches, in the Municipality \\ of Peruíbe, São Paulo State, Brazil \\ Nayla de Jesus Zanella Carramão' (iD, Marisa Cristina de Almeida Guimarães² \\ ' Superintendência de Controle de Endemias, Programa de Aprimoramento Profissional, Centro Regional de São Vicente, Laboratório de Malacologia, \\ São Vicente, São Paulo, Brasil \\ 2 Superintendência de Controle de Endemias, Centro Regional de São Vicente, Laboratório de Malacologia, São Vicente, São Paulo, Brasil
}

\begin{abstract}
RESUMO
OBJETIVOS: Identificar moluscos dulciaquícolas e larvas de trematódeos oriundos de valas de drenagem no município de Peruíbe, estado de São Paulo, Brasil, e descrever a morfologia das larvas emergidas. MATERIAIS E MÉTODOS: Moluscos foram coletados em 53 valas. No laboratório, procedeu-se à identificação morfológica dos espécimes e pesquisas parasitológicas para determinação e descrição morfológica das larvas. RESULTADOS: Foram coletados 5.969 moluscos pertencentes às famílias Planorbidae, Lymnaeidae, Physidae, Thiaridae e Ampullariidae. Os exames parasitológicos revelaram nove formas larvais correspondentes a sete morfotipos: xifidiocercária, equinocercária, estrigeocercária, distoma brevifurcada faringeada, distoma brevifurcada afaringeada, pleurolofocercária e anfistomocercária. A espécie Biomphalaria tenagophila (d'Orbigny, 1835) foi suscetível a oito cercárias das nove encontradas; e seis exemplares de moluscos estavam parasitados por larvas de Schistosoma mansoni Sambon, 1907. CONCLUSÃO: É fundamental estabelecer programas de controle e vigilância malacológica em áreas vulneráveis quando moluscos hospedeiros intermediários de parasitas de importância médica e veterinária colonizam esses ambientes, sendo especialmente importante quando esses moluscos estão infectados com larvas de S. mansoni.
\end{abstract}

Palavras-chave: Cercárias; Moluscos; Esquistossomose; Trematódeos.

\begin{abstract}
OBJECTIVES: Identify freshwater mollusks and trematode larvae from drainage ditches in the Municipality of Peruíbe, São Paulo State, Brazil, and describe the morphology of emerged larvae. MATERIALS AND METHODS: Mollusks were collected from 53 ditches. In the laboratory, the specimens were morphologically identified, and parasitological analysis was carried out to determine and describe the larvae. RESULTS: 5,969 mollusks belonging to the families Planorbidae, Lymnaeidae, Physidae, Thiaridae, and Ampullariidae were collected. The parasitological analysis of the mollusks revealed nine different larvae corresponding to the following seven types of cercariae: xiphidio cercariae, echinostome cercariae, strigea cercariae, brevifurcate pharyngeate distome cercariae, brevifurcate apharyngeate distome cercariae, pleurolophocercous cercariae, and amphistome cercariae. Biomphalaria tenagophila (d'Orbigny, 1835) was susceptible to eight of the nine cercariae found, and six specimens of mollusks were parasitized by Schistosoma mansoni Sambon, 1907. CONCLUSION: It is fundamental to establish malacological control and surveillance programs in vulnerable areas when mollusks, intermediate host of parasites of medical and veterinary importance, colonize these environments, especially when infected with $S$. mansoni larvae.
\end{abstract}

Keywords: Cercariae; Mollusks; Schistosomiasis; Trematodes.

\footnotetext{
Correspondência / Correspondence:

Marisa Cristina de Almeida Guimarães

Superintendência de Controle de Endemias, Centro Regional de São Vicente, Laboratório de Malacologia

Rua João Ramalho, 587. Bairro: Centro. CEP: $11310-050$ - São Vicente, São Paulo, Brasil - Tel.: + 55 (13) 3468-8894

E-mail: mcag.biologa@gmail.com
} 


\section{INTRODUÇÃO}

Os moluscos gastrópodes dulciaquícolas possuem representantes em várias famílias de importância médica e veterinária, sendo encontrados em diversos tipos de coleções hídricas'. As principais famílias desses moluscos são Planorbidae, Lymnaeidae, Physidae, Thiaridae, Ampullariidae, Ancylidae e Hydrobiidae que desempenham papel de hospedeiros intermediários dos trematódeos digenéticos ${ }^{1,2}$.

Entre os planorbídeos, o gênero Biomphalaria Preston, 1910 se destaca no Brasil com 10 espécies descritas e uma subespécie. Oito espécies foram identificadas no estado de São Paulo, sendo três hospedeiras naturais do trematódeo Schistosoma mansoni Sambon, 1907, Biomphalaria glabrata (Say, 1818), Biomphalaria straminea (Dunker, 1848) e Biomphalaria tenagophila (d'Orbigny, 1835); uma espécie potencial hospedeira do S. mansoni, Biomphalaria peregrina (d'Orbigny, 1835); e quatro espécies não hospedeiras do S. mansoni, Biomphalaria occidentalis (Paraense, 1981), Biomphalaria intermedia (Paraense \& Deslandes, 1962), Biomphalaria oligoza (Paraense, 1975) e Biomphalaria schrammi (Crosse, $1984)^{1,2,3}$.

Com relação à esquistossomose no estado de São Paulo, a doença possui um perfil epidemiológico de baixa prevalência, porém com grandes áreas de transmissão autóctone e de casos importados, os quais compreendem as regiões do Vale do Ribeira de Iguape, Baixada Santista, Região Metropolitana de Campinas, Região Metropolitana de São Paulo, Vale do Paraíba e Litoral Norte ${ }^{4,5}$.

As doenças causadas por trematódeos são transmitidas por penetração direta da larva na pele e transmissão alimentar ${ }^{6}$. No primeiro caso, por exemplo, as larvas do S. mansoni e, no segundo caso, pela ingestão de água e de alimentos como peixes e crustáceos crus ou malcozidos, hortaliças cruas e mal lavadas, podendo resultar em várias doenças, como a equinostomíase, paragonimíases, clonorquíase, dermatites cercarianas em humanos e fasciolíase $7,8,9,10$.

Os estudos desenvolvidos sobre as interações parasitas-hospedeiros são baseados em levantamentos faunísticos considerando o sistema moluscotrematódeo. Esse modelo de investigação é muito utilizado nas pesquisas no Brasil e em outros países $^{11,12,13}$

Apesar dos conhecimentos adquiridos, é necessário elucidar diversas larvas e associá-las às formas adultas, bem como conhecer aspectos relacionados ao ciclo biológico e outros. Somente assim, será possível interpretar corretamente os papéis que os hospedeiros intermediários cumprem na transmissão das doenças ${ }^{14,15}$.

$\bigcirc$ presente estudo tem como objetivo identificar moluscos dulciaquícolas e larvas de trematódeos procedentes de valas de drenagem no município de Peruíbe, estado de São Paulo, e descrever a morfologia das larvas eliminadas pelos moluscos.

\section{MATERIAIS E MÉTODOS}

\section{ÁREAS DE ESTUDO}

As coletas dos moluscos foram realizadas nos meses de outubro a dezembro de 2016 e de abril a julho de 2017, em valas de irrigação no peridomicílio de três áreas do município de Peruíbe, litoral sul do estado de São Paulo (Figura 1). As áreas selecionadas foram: A Nova Peruíbe: $24^{\circ} 17^{\prime} 50^{\prime \prime} S, 47^{\circ} 1^{\prime} 24^{\prime \prime} \mathrm{W}$ (área periférica) com 14 valas; B - Jardim Caraguava: 241 7'42"S, 4700'56"W (área periférica) com 28 valas; e C Ruínas: 241 6'8"S, 4656'12"W (área urbana) com 11 valas. Todas as áreas foram georreferenciadas com a utilização do aparelho GPS (global position system) eTrex Summit ${ }^{\circledR}$ (Garmin).

A área C (Ruínas) foi selecionada em razão das semelhanças ambientais com as áreas A (Nova Peruíbe) e B (Jardim Caraguava), como a presença de valas de irrigação com água e vegetação no peridomicílio, assim como larvas de insetos, girinos, moluscos e alguns peixes. No entorno das valas, foram notados alguns animais como cães, gatos, aves (urubus, patos, galinhas) e eventualmente cavalos. Todas as áreas tinham saneamento básico; no entanto, havia efluentes sanitários sendo lançados nas valas, contaminando também os solos pela ausência de pavimentação.

\section{COLETA DE MOLUSCOS}

Para a realização das coletas, não foi necessário solicitar licença ao Instituto Brasileiro do Meio Ambiente e dos Recursos Naturais Renováveis, em razão da Superintendência de Controle de Endemias (Sucen) ser responsável pelas ações de vigilância e controle de vetores e hospedeiros intermediários ${ }^{1}$.

A metodologia empregada nas coletas seguiu - Manual da Sucen: Procedimento Operacional Padrão para o Programa de Vigilância e Controle da Esquistossomose Mansônica (POPESQUISTO) ${ }^{16}$. As coletas dos moluscos ocorreram em toda a extensão da vala, coletando-se três amostras a cada trecho de $100 \mathrm{~m}$.

Considerou-se, como amostra, um copo de plástico resistente (volume de $200 \mathrm{~mL}$ ), com capacidade para 20 a 30 moluscos aproximadamente, conforme o tamanho do animal. Os copos utilizados receberam um pouco de vegetação aquática para manutenção da umidade interna, cuja abertura foi vedada com gaze. As amostras foram identificadas quanto à data da coleta, número da amostra, nome da localidade (bairro), nome da vala (igual ao nome da rua) e número do trecho da vala.

As coletas foram realizadas com o auxílio de uma pá coletora, constituída de tela metálica (malha de $2 \mathrm{~mm}$ ) em ângulo reto, com o cabo de madeira longo e uma pinça de ponta reta de aço inox de $30 \mathrm{~cm}$. Ao final das coletas, todas as amostras foram acondicionadas em caixas de plástico para serem transportadas com segurança. 


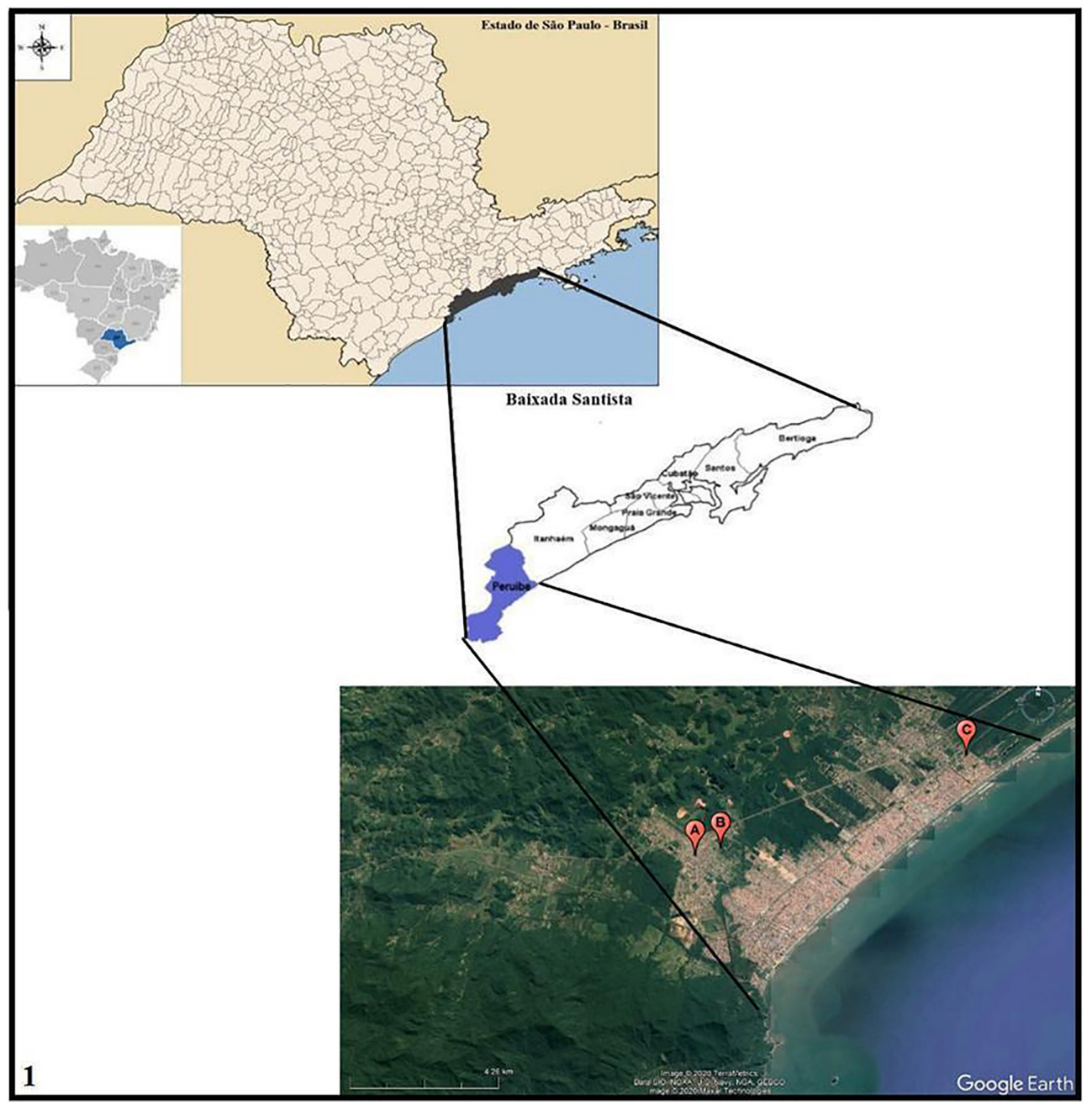

Fonte: Tabwin versão 4.1.5 - Datasus; Google Earth. Imagem produzida por Nayla Zanella Carramão. Área A: Nova Peruíbe; Área B: Jardim Caraguava; Área C: Ruínas.

Figura 1 - Localização de Peruíbe na Baixada Santista, estado de São Paulo, Brasil, e imagem via satélite das áreas de coletas malacológicas

\section{TRATAMENTO DOS MOLUSCOS}

Os procedimentos iniciais aconteceram no período da tarde, assim que as amostras do campo chegavam ao laboratório. Os moluscos foram quantificados e separados por gênero, segundo os parâmetros conquiológicos ${ }^{1}$. Após a triagem, os animais seguiram agrupados de acordo com o gênero ao qual pertenciam. Cada recipiente recebeu até 15 moluscos, água isenta de cloro e pequena porção de alface fresca.

\section{EXAME PARASITOLÓGICO}

Os exames para a avaliação da infecção por cercárias nos moluscos ocorreram individualmente em alvéolos da placa de cultura com água, exceto animais de maior diâmetro que foram colocados em pequenos recipientes de vidro transparente $(7 \mathrm{~cm})$. Os moluscos foram expostos à luz artificial (fotoestimulação) por $2 \mathrm{~h}$, sempre no período diurno.

As formas larvais que emergiram dos moluscos foram observadas na própria placa de cultura de célula, que foi colocada sobre a base do microscópio estereoscópico em aumentos de 10-15x. Para um maior detalhamento das estruturas larvais, foi necessário colocar as larvas entre lâmina e lamínula para serem analisadas no microscópio óptico.

As pesquisas parasitológicas foram finalizadas com o esmagamento dos moluscos, visando encontrar possíveis cercárias em seus tecidos internos (vísceras). Para tanto, foi utilizada uma placa de vidro de $8,0 / 12,0 \mathrm{~cm}$ que recebeu até cinco moluscos, conforme o tamanho do animal; e, com duas placas, realizou-se o esmagamento da concha, por meio de uma leve pressão ${ }^{16,17}$. As taxas de infecção global e específica dos moluscos foram determinadas após os exames, segundo o método descrito por Ruiz ${ }^{18}$.

\section{PREPARAÇÃO DAS LÂMINAS}

As larvas encontradas na água e nas vísceras dos moluscos esmagados foram capturadas com pipeta e transferidas para lâminas com uma gota de água para serem examinadas a fresco e, posteriormente, com os corantes lugol $(0,1 \%)$ e vermelho neutro $(0,05 \%)$, foram levadas ao microscópio óptico. A fotografia das larvas ocorreu em aumentos de 40x, exceto para Apharyngostrigea sp., larva mais robusta, que foi fotografada em aumento de 10x. 
A identificação morfológica das larvas foi baseada nos seguintes critérios: formato do corpo e da cauda; tamanho e posição das ventosas; presença ou ausência de estruturas especializadas, como estilete oral e ocelos; presença de faringe; entre outras estruturas, conforme mostram as chaves taxonômicas de Pinto e Melo9, Martorelli et al. ${ }^{15}$, Naruto ${ }^{19}$, Frandsen e Christensen ${ }^{20}$, Shell ${ }^{21}$ e Boaventura et al. ${ }^{22}$.

\section{IDENTIFICAÇÃO DE BIOMPHALARIA SPP.}

Após os exames parasitológicos iniciais, foram separados dois ou três exemplares de Biomphalaria spp. que não liberaram cercárias para a identificação das espécies, conforme os protocolos do Ministério da Saúde', de Deslandes ${ }^{23}$, da Pan American Health Organization $^{24}$ e de Paraense ${ }^{25}$.

A identificação e as imagens foram feitas no Laboratório de Malacologia do Centro Regional de São Vicente/Sucen.

\section{RESULTADOS}

\section{MOLUSCOS COLETADOS E IDENTIFICADOS}

Foram coletados e examinados 5.969 moluscos, procedentes de 53 valas distribuídas nas três áreas:
1.163 moluscos na área $A ; 3.841$ moluscos na $B$; e 965 moluscos na C. A identificação dos moluscos revelou B. tenagophila, B. straminea, Drepanotrema sp. (Planorbidae), Lymnaea sp. (Lymnaeidae), Physa sp. (Physidae), Pomacea sp. (Ampullariidae) e Melanoides tuberculata (Müller, 1774) (Thiaridae) (Tabela 1).

\section{IDENTIFICAÇÃO DE TREMATÓDEOS E TAXAS DE INFECÇÃO}

Nove formas larvais foram registradas e classificadas em sete tipos morfológicos, a saber: xifidiocercária - Cercaria lutzi (Ruiz, 1943); equinocercária - Cercaria granulifera (Lutz, 1924) e Echinostoma sp.; estrigeocercária - Cercaria caratinguensis (Ruiz, 1953) e Apharyngostrigea sp.; distoma brevifurcada faringeada - Cercaria ocellifera (Lutz, 1917); distoma brevifurcada afaringeada Cercaria blanchardi (Pirajá da Silva, 1912) ou larva de S. mansoni; pleurolofocercária - Centrocestus formosanus (Nishigori, 1924); e anfistomocercária - Anfistoma sp. As larvas C. lutzi, C. granulifera, C. caratinguensis e C. ocellifera apareceram com maior frequência (Tabela 2).

Tabela 1 - Frequência de moluscos dulciaquícolas coletados em três áreas de Peruíbe, estado de São Paulo, Brasil, 2016-2017

\begin{tabular}{|c|c|c|c|}
\hline Moluscos & $\begin{array}{c}\text { Área A } \\
\mathrm{N}\end{array}$ & $\begin{array}{c}\text { Área B } \\
\quad \mathrm{N}\end{array}$ & $\begin{array}{c}\text { Área C } \\
\quad \mathrm{N}\end{array}$ \\
\hline Biomphalaria tenagophila & 962 & 3.592 & 631 \\
\hline Biomphalaria straminea & - & - & 4 \\
\hline Melanoides tuberculata & 19 & 4 & 163 \\
\hline Physa sp. & 169 & 232 & 124 \\
\hline Lymnaea sp. & - & 6 & 39 \\
\hline Drepanotrema sp. & - & 3 & - \\
\hline Pomacea sp. & 13 & 4 & 4 \\
\hline Total & 1.163 & 3.841 & 965 \\
\hline
\end{tabular}

Área A: Nova Peruíbe; Área B: Jardim Caraguava; Área C: Ruínas. Sinal convencional utilizado: - Dado numérico igual a zero, não resultante de arredondamento.

Tabela 2 - Frequência de moluscos infectados, segundo formas larvais e taxa de infecção por área pesquisada, no município de Peruíbe, estado de São Paulo, Brasil, 2016-2017

\begin{tabular}{|c|c|c|c|c|c|c|c|}
\hline \multirow{2}{*}{ Formas larvais } & \multirow{2}{*}{ Moluscos infectados } & \multicolumn{2}{|c|}{ Área A } & \multicolumn{2}{|c|}{ Área B } & \multicolumn{2}{|c|}{ Área C } \\
\hline & & N & $\%$ & $\mathrm{~N}$ & $\%$ & $N$ & $\%$ \\
\hline \multirow{2}{*}{ Cercaria lutzi } & Biomphalaria tenagophila & 9 & 0,93 & 324 & 9,02 & 16 & 2,53 \\
\hline & Physa sp. & 1 & 0,59 & - & - & - & - \\
\hline Cercaria granulifera & Biomphalaria tenagophila & 4 & 0,41 & 59 & 1,64 & 25 & 3,96 \\
\hline Echinostoma sp. & Biomphalaria tenagophila & - & - & 1 & 0,02 & - & - \\
\hline Cercaria caratinguensis & Biomphalaria tenagophila & 4 & 0,41 & 38 & 1,05 & - & - \\
\hline Apharyngostrigea sp. & Biomphalaria tenagophila & - & - & - & - & 3 & 0,47 \\
\hline Cercaria ocellifera & Biomphalaria tenagophila & - & - & 9 & 0,25 & 2 & 0,31 \\
\hline Cercaria blanchardi & Biomphalaria tenagophila & - & - & 6 & 0,16 & - & - \\
\hline Centrocestus formosanus & Melanoides tuberculata & 1 & 5,26 & - & - & - & - \\
\hline Anfistoma sp. & Biomphalaria tenagophila & - & - & 1 & 0,02 & - & - \\
\hline
\end{tabular}

Área A: Nova Peruíbe; Área B: Jardim Caraguava; Área C: Ruínas. Sinal convencional utilizado: - Dado numérico igual a zero, não resultante de arredondamento. 
Entre os espécimes de B. tenagophila coletados na área B (Jardim Caraguava), seis exemplares portavam larvas de S. mansoni, representando uma taxa de infecção de 0,16\%. Entretanto, as maiores taxas de infecção de B. tenagophila foram com C. lutzi (9,02\%), na área $B$, e C. granulifera (3,96\%), na área C (Ruínas). As taxas de infecção dos moluscos, quando comparadas por área pesquisada, resultaram em $11,40 \%, 4,76 \%$ e 1,63\%, respectivamente, para Jardim Caraguava, Ruínas e Nova Peruíbe (Tabela 2).

\section{DESCRIÇÃO MORFOLÓGICA DAS LARVAS ENCONTRADAS} NOS EXAMES PARASITOLÓGICOS

Duas larvas distintas do tipo equinocercária (Figura 2: A e D) foram observadas. Uma delas, denominada de Echinostoma sp., apresentou: membrana natatória na cauda simples (Figura 2: B); ventosas oral e ventral de tamanhos semelhantes; numerosas células cistogênicas espalhadas pelo corpo; presença de esôfago e dois cecos intestinais longos, os quais seguem para a região posterior do corpo (Figura 2: A). Larvas produzidas em rédias de coloração acastanhada (Figura 2: C).

A segunda equinocercária (Figura 2: D e E) apresentou: faringe muscular, esôfago longo e bifurcado em dois cecos intestinais, seguindo para a região posterior do corpo; vesículas excretoras em cada lado do corpo, com canais coletores que possuem, nas porções mais dilatadas, 35-45 granulações esféricas refringentes ou concreções de fácil visualização; ventosa oral e ventral; presença de dois grânulos refringentes situados à frente da faringe e próximos à base da ventosa oral (Figura 2: E); cauda simples, longa e estreita recoberta por espinhos. Tais características são de C. granulifera, forma larval de Paryphostomum segregatum (Dietz, 1909). Desenvolvimento em rédias ${ }^{8}$ (Figura 2: F).

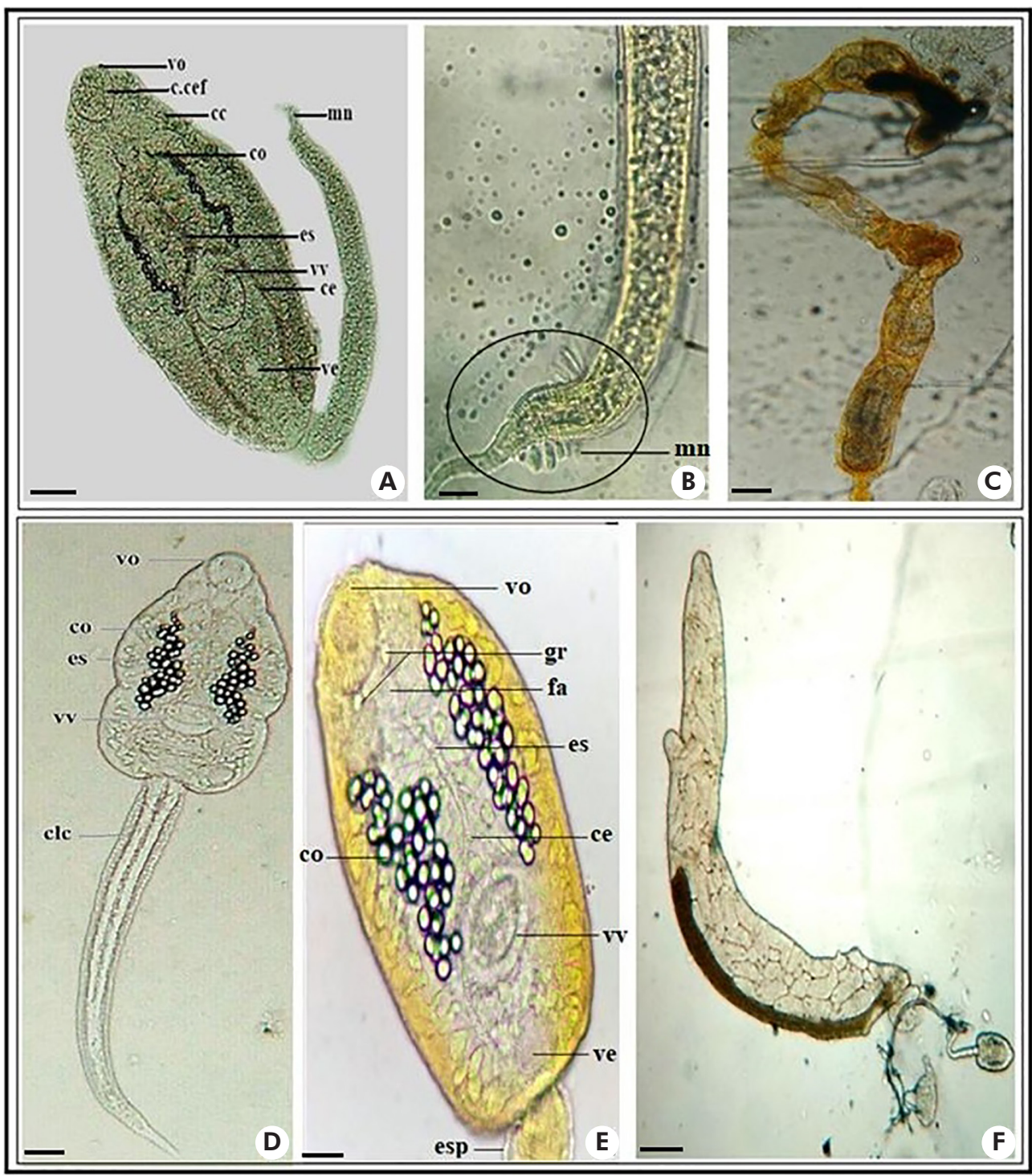

Fotos: Nayla Zanella Carramão.

A: Corpo e cauda de Echinostoma sp.; B: Membrana natatória de Echinostoma sp.; C: Rédia acastanhada de Echinostoma sp.; D: Corpo e cauda de Cercaria granulifera; E: Estruturas do corpo e cauda da Cercaria granulifera; F: Rédia acastanhada de Cercaria granulifera. Corante utilizado - Lugol diluído 0,1\% (A, B, E); Sem corante - a fresco (D, F). Escala: $50 \mu \mathrm{m}$. vo: Ventosa oral; fa: Faringe; gr: Grânulos; c.cef: Colar cefálico; cc: Células cistogênicas; co: Concreções; es: Esôfago; vv: Ventosa ventral; ce: Cecos; ve: Vesícula excretora; mn: Membrana natatória; clc: Células caudais; esp: Espinhos.

Figura 2 - Larvas do tipo equinocercária 
Uma larva do tipo xifidiocercária, C. lutzi (Figura 3: $A$ e B), foi encontrada apresentando: ventosa oral bem desenvolvida e estilete, e ventosa ventral na região mediana do corpo; presença de glândulas de penetração facilmente observadas, sendo cinco em cada lado do corpo; vesícula excretora em forma de "Y" e cauda simples. Desenvolvimento em esporocistos (Figura 3: C).

Duas larvas do tipo estrigeocercária (Figura 3: D e E) foram encontradas, sendo uma delas semelhante a C. caratinguensis, com: corpo alongado; ventosas oral e ventral, sendo a ventosa ventral localizada na porção média do corpo; ocelos sem pigmentação; cauda longifurcada, com furcas achatadas lateralmente e mais longas que a haste, sendo que, em seu interior, foram observados cinco pares de células. Desenvolvimento em esporocistos ${ }^{20,21}$.

A segunda estrigeocercária (Figura 3: E) apresentou características semelhantes à larva Apharyngostrigea: corpo grande e oval; presença de ventosas oral e ventral (bem esférica); uma faringe; duas glândulas de penetração em formato de bolsa de cada lado do corpo, logo abaixo da ventosa oral; presença do primórdio genital no corpo próximo ao início da cauda; cauda bifurcada, com furcas médias e largas, achatadas dorso ventralmente, um tronco caudal acentuadamente globoso; presença de grânulos de cor marrom no interior do corpo e da cauda. Desenvolvimento em esporocistos ${ }^{20,21}$.

As larvas do tipo distoma brevifurcada faringeada (Figura 4: A e B) assemelham-se com C. ocellifera, pertencente à família Clinostomidae, com: membrana dorsal no corpo alongado; ocelos pigmentados; ventosa oral grande e ovalada, ventosa ventral não visualizada; cauda longa e bifurcada em furcas curtas. Desenvolvimento em rédias ${ }^{8}$.

A larva do tipo anfistomocercária (Figura 4: E) apresentou: corpo alongado, com células cistogênicas espalhadas e presença de dois ocelos pigmentados; ventosas oral e ventral grandes, estando a ventosa ventral localizada na região posterior do corpo; cauda simples. Desenvolvimento em rédias ${ }^{8,26}$.

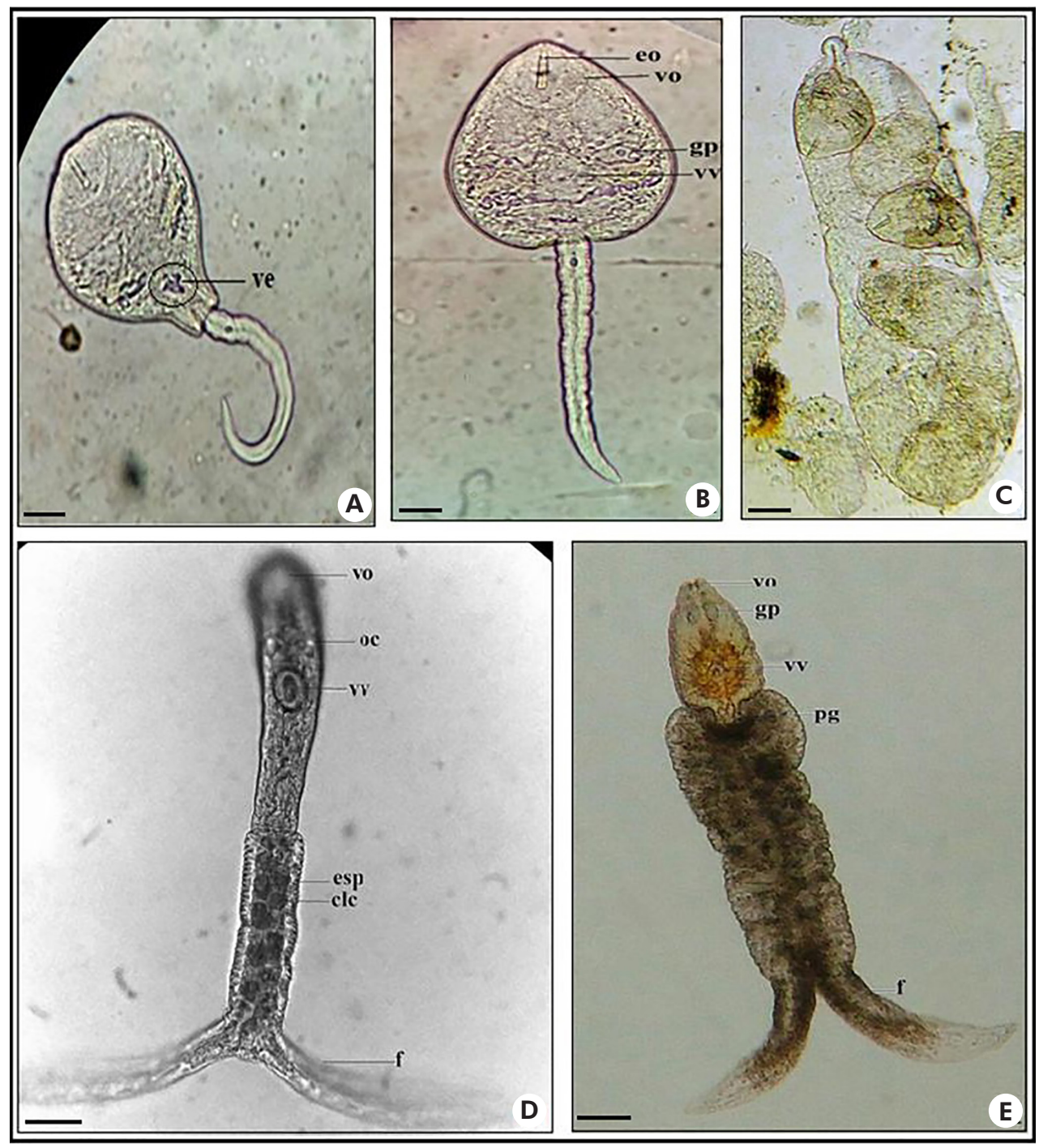

Fotos: Nayla Zanella Carramão.

A, B: Corpo e cauda de Cercaria lutzi; C: Esporocistos de Cercaria lutzi; D: Corpo e cauda de Cercaria caratinguensis; E: Corpo e cauda de Apharyngostrigea sp. Exame a fresco. Escala: $50 \mu \mathrm{m}$. ve: Vesícula excretora (Y); eo: Estilete oral; vo: Ventosa oral; gp: Glândulas de penetração; vv: Ventosa ventral; oc: Ocelos; esp: Espinhos; clc: Células caudais; f: Furca; pg: Primórdio genital.

Figura 3 - Larvas do tipo xifidiocercária $(A, B, C)$ e estrigeocercária $(D, E)$ 


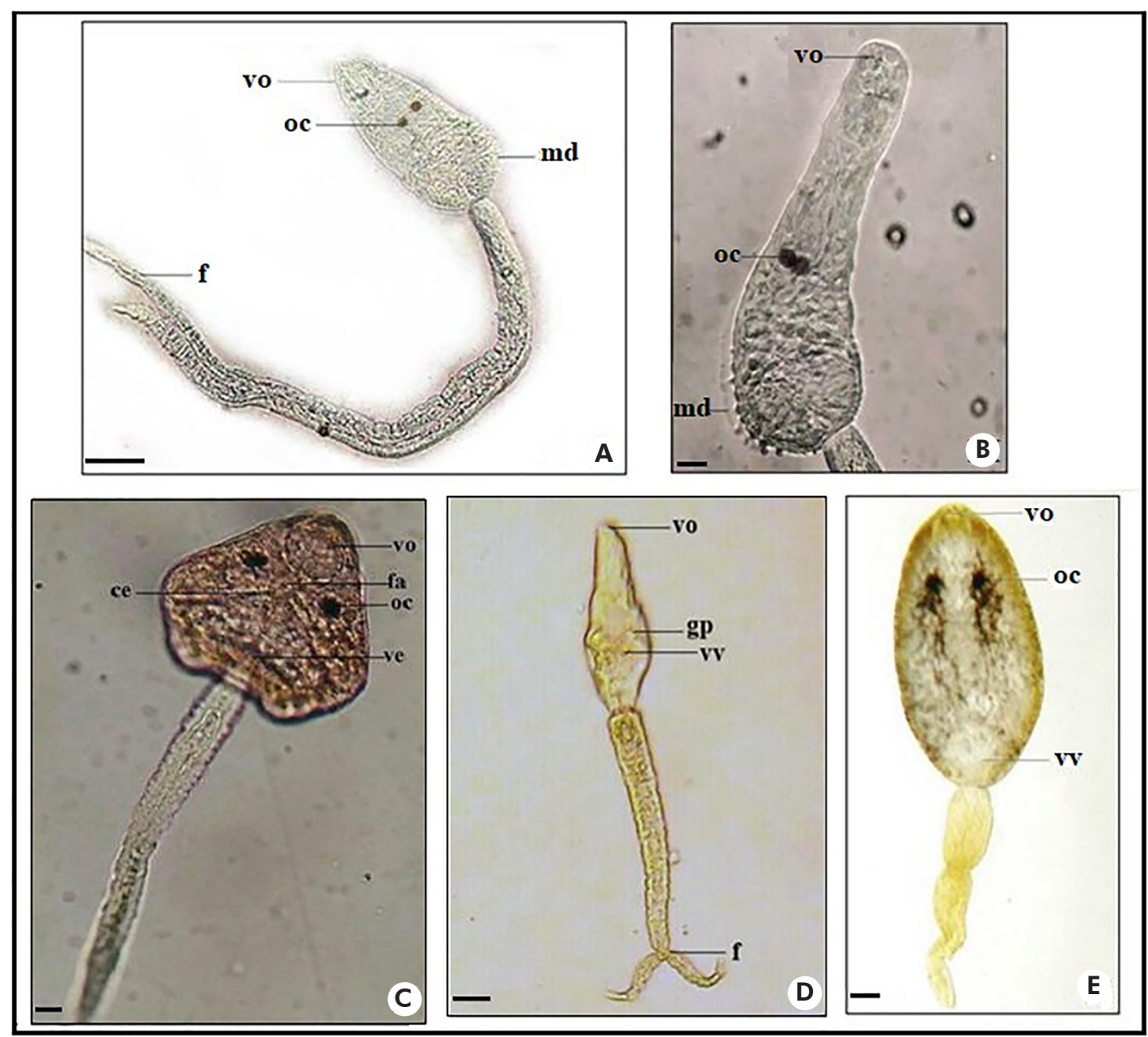

Fotos: Nayla Zanella Carramão

A: Corpo e cauda de Cercaria ocellifera; B: Corpo de Cercaria ocellifera; C: Corpo e cauda de Centrocestus formosanus; D: Corpo e cauda de Cercaria blanchardi (Schistosoma mansoni); E: Corpo e cauda de Anfistoma sp. Corantes: vermelho neutro 0,05\% (C) e lugol 0,1\% (D, E). Escala: 50 m. vo: Ventosa oral; oc: Ocelos; md: Membrana dorsal; f: Furca; vv: Ventosa ventral; fa: Faringe; ce: Cecos; ve: Vesícula excretora; gp: Glândulas de penetração.

Figura 4 - Larvas do tipo distoma brevifurcada faringeada, pleurolofocercária e anfistomocercária - com ocelos pigmentados $(A, B, C, E)$; e distoma brevifurcada afaringeada-sem ocelos pigmentados (D)

A larva do tipo pleurolofocercária (Figura 4: C) apresentou características de C. formosanus com: corpo oval e numerosas células cistogênicas; ventosa oral grande, e ventosa ventral de difícil visualização; faringe muscular; dois cecos intestinais longos, abaixo dos ocelos e vesícula excretora na parte proximal da cauda; cauda simples com pequena membrana natatória. Desenvolvimento em rédias ${ }^{27,28}$

A larva do tipo distoma brevifurcada afaringeada, representada por C. blanchardi (Figura 4: D), apresentou: corpo alongado; ventosa oral desenvolvida, e ventosa ventral pequena, localizada na região subequatorial do corpo; glândulas de adesão e penetração; faringe ausente; cauda cilíndrica, bifurcada em furcas curtas. Desenvolvimento em esporocistos ${ }^{19}$.

\section{DISCUSSÃO}

Os resultados obtidos permitiram identificar as formas larvais e os moluscos hospedeiros intermediários dos trematódeos, ampliando o conhecimento sobre as condições epidemiológicas e sanitárias dos locais pesquisados em Peruíbe. Ressalta-se que, apesar de existir saneamento básico, as valas recebiam efluentes sanitários, favorecendo a transmissão de doenças e possivelmente a esquistossomose.

As pesquisas malacológicas resultaram em 5.969 moluscos coletados. A espécie B. tenagophila predominou em todas as áreas, assim como Physa sp. foi a segunda numericamente superior. É importante considerar que a fauna malacológica possui estreita relação com os fatores ambientais, e alguns grupos taxonômicos podem ser mais sensíveis a determinadas perturbações ambientais que outros ${ }^{9}$. Por essas características biológicas, os moluscos são muito utilizados nas avaliações e monitoramentos das condições ambientais $^{12,13}$.

Quando comparada globalmente, a taxa de infecção foi maior no Jardim Caraguava (área B), com $11,4 \%$. Em relação à taxa de infecção específica, B. tenagophila foi a espécie mais parasitada e suscetível a oito das nove formas larvais encontradas (Tabela 2). Echinostoma sp., Apharyngostrigea sp., C. formosanus e Anfistoma sp. foram eliminadas por poucos moluscos. Esses dados podem indicar a influência de fatores ambientais sobre as larvas de trematódeos; 
entretanto, tais aspectos podem ser elucidados com novos estudos.

Os estudos morfológicos revelaram seis espécies de larvas de trematódeos, e três larvas não receberam a identificação específica, como Echinostoma sp., Apharyngostrigea sp. e Anfistoma sp. (Tabela 2). Segundo Locke et al. ${ }^{29}$, as espécies não podem ser identificadas apenas pela morfologia. É necessário combinar com as ferramentas moleculares nos estágios larvais associados aos adultos conhecidos.

Em relação à C. lutzi (Figura 3: $A$ e B), essa larva foi associada ao parasito Pneumonoeces neivai (Travassos \& Artigas, 1927) encontrado em pulmão de anuros ${ }^{30,31}$. Os trematódeos da família Echinostomatidae são parasitas intestinais de aves, répteis e mamíferos, inclusive humanos, com relatos de casos na Ásia9,14. A equinocercária identificada como C. granulifera (Figura 2: D e E) foi considerada a forma larval de $P$. segregatum. Ruiz ${ }^{32}$ verificou as formas císticas na faringe de girinos, peixes e moluscos e a forma adulta do parasita no urubu.

A larva Anfistoma sp. (Figura 4: E), do grupo Paramphistomoidea, tem importância veterinária, principalmente para ruminantes ${ }^{33}$. A larva Anfistoma lunatum foi associada ao parasita Zygocotyle lunata (Diesing, 1836), encontrado em mamíferos ruminantes e em aves, como a galinha caipira (Gallus gallus domesticus). De acordo com Barbosa ${ }^{26}$, o encistamento da larva ocorre em substratos do meio externo, como concha de molusco, crustáceo, tegumento de girinos, vegetação ou qualquer objeto.

C. formosanus (Figura 4: C) foi associada à centrocestíase em humanos na Ásia (Taiwan e Filipinas), adquirida pelo consumo de peixes crus com metacercárias ${ }^{26,33,34}$. As larvas identificadas como C. caratinguensis e Apharyngostrigea sp. (Figura 3: $\mathrm{D}$ e E) pertencem à família Strigeidae. Os parasitos foram encontrados em aves e mamíferos e as metacercárias em peixes, moluscos e anfíbios ${ }^{35}$.

C. ocellifera (Figura 4: A e B) é considerada a forma larval de Clinostomum heluans Braun, 1989, da família
Clinostomatidae, parasita de répteis e aves aquáticas, também encontrado em girinos, peixes e moluscos, seus hospedeiros intermediários ${ }^{31}$.

Por fim, salienta-se o encontro de larvas de S. mansoni (C. blanchardi) em seis espécimes de B. tenagophila procedentes de valas do Jardim Caraguava, área afetada por enchentes. Moradores relatam que, nas ocasiões de enchentes, os moluscos são carreados para dentro das casas, sendo inevitável - contato físico, demonstrando ser essa mais uma situação de vulnerabilidade ambiental que pode propiciar a transmissão de várias doenças, entre elas, a esquistossomose. Segundo Artigas et al. ${ }^{36}$, casos de esquistossomose são reportados em Peruíbe desde 1966.

\section{CONCLUSÃO}

A presença de larvas de trematódeos na área estudada indica a existência de focos ativos que requerem atenção especialmente pela presença de moluscos portando larvas de S. mansoni. Assim, medidas de controle e vigilância malacológica são imprescindíveis em áreas vulneráveis como a de Peruíbe, cuja fauna de moluscos é composta por famílias de importância médica e veterinária.

\section{AGRADECIMENTOS}

À equipe de campo, pelo excelente trabalho; e aos técnicos do Laboratório de Malacologia, que colaboraram com a presente pesquisa, em especial ao Antonio da Silva.

\section{CONFLITOS DE INTERESSES}

Não há conflitos de interesse associados a este estudo.

\section{CONTRIBUIÇÃO DOS AUTORES}

Os autores participaram de todas as etapas do estudo, ou seja, concepção e delineamento, execução (campo e laboratório), análises e produção do manuscrito.

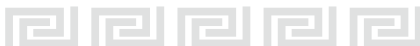

\section{REFERÊNCIAS}

1 Ministério da Saúde (BR). Secretaria de Vigilância em Saúde. Departamento de Vigilância Epidemiológica. Vigilância e controle de moluscos de importância epidemiológica: diretrizes técnicas: Programa de Vigilância e Controle da Esquistossomose (PCE). 2. ed. Brasília: Ministério da Saúde; 2008. (Série A. Normas e manuais técnicos)

2 Ohlweiler FP, Takahashi FY, Guimarães MCA, Gomes SR, Kawano T. Manual de gastrópodes límnicos e terrestres do Estado de São Paulo associados às helmintoses. Porto Alegre: Redes; 2010.
3 Palasio RGS, Guimarães MCA, Ohlweiler FP, Tuan R. Molecular and morphological identification of Biomphalaria species from the state of São Paulo, Brazil. ZooKeys. 2017 Apr;668:11-32.

4 Secretaria de Estado da Saúde (SP). Centro de Vigilância Epidemiológica "Prof. Alexandre Vranjac. Esquistossomose [Internet]. São Paulo; 2021 [citado 2021 jan 12]. Disponível em: https://www.saude. sp.gov.br/resources/cve-centro-de-vigilancia-epid emiologica/areas-de-vigilancia/doencas-de-tran smissao-por-vetores-e-zoonoses/dados/esquisto/ esquisto_dados.pdf. 
5 Secretaria de Estado da Saúde (SP). Centro de Vigilância Epidemiológica "Prof. Alexandre Vranjac. Esquistossomose mansoni no Estado de São Paulo: novas estratégias de controle e critérios para eliminação da transmissão [Internet]. São Paulo; 2020 [citado 2020 abr 7]. Disponível em: http:// www.saude.sp.gov.br/resources/cve-centro-devigilancia-epidemiologica/areas-de-vigilancia/ doencas-transmitidas-por-agua-e-alimentos/ eventos/2esquil 1 _estrategias.pdf.

6 Rey L. Bases da parasitologia médica. 3. ed. Rio de Janeiro: Guanabara Koogan; 2010.

7 Farahnak A, Vafaie-Darian R, Mobedi I. A faunistic survey of cercariae from fresh water snails: Melanopsis spp. and their role in disease transmission. Iranian J Publ Health. 2006;35(4):70-4.

8 Pinto HA. Biologia e taxonomia de trematódeos transmitidos por moluscos dulciaquícolas na represa da Pampulha, Belo Horizonte, Minas Gerais, Brasil [tese]. Belo Horizonte (MG): Universidade Federal de Minas Gerais, Instituto de Ciências Biológicas; 2013. 299 p.

9 Pinto HA, Melo AL. Larvas de trematódeos em moluscos no Brasil: panorama e perspectivas após um século de estudos. Rev Patol Trop. 2013 out/ dez;42(4):369-86.

10 Neves DP, coordenador. Parasitologia humana. 13. ed. São Paulo: Atheneu; 2016.

11 Madsen H, Hung NM. Reprint of "An overview of freshwater snails in Asia with main focus on Vietnam". Acta Trop. 2015 Jan;1 41 (P+ B):372-84.

12 Soldánová M, Selbach C, Sures B, Kostadinova A, Pérez-del-Olmo A. Larval trematode communities in Radix auricularia and Lymnaea stagnalis in a reservoir system of the Ruhr River. Parasit Vectors. 2010 Jun;3:56.

13 Dodangeh S, Daryani A, Sharif M, Gholami S, Kialashaki $E$, Moosazadeh $M$, et al. Freshwater snails as the intermediate host of trematodes in Iran: a systematic review. Epidemiol Health. 2019;41:e2019001.

14 Souza MAA, Melo AL. Caracterização de larvas de trematódeos emergentes de moluscos gastrópodes coletados em Mariana, Minas Gerais, Brasil. Iheringia, Ser Zool. 2012 mar; 102(1):1 1-8.

15 Martorelli SR, Alda P, Marcotegui P, La Sala LF, Montes MM. Larval digeneans in Biomphalaria snails from the Salto Grande Dam area in the Uruguay River. Publicacion del Laboratorio de Helmintos y Parasitos de Crustaceos del Cepave; 2013. 13 p.

16 Secretaria de Estado da Saúde (SP). Superintendência de Controle de Endemias. Manual de procedimento operacional padrão para o Programa de Vigilância e Controle da Esquistossomose Mansônica: POPESQUISTO. São Paulo; 2018.
17 Carvalho OS, Passos LKJ, Mendonça CLF, Cardoso PCM, Caldeira RL. Moluscos brasileiros de importância médica. 2. ed. Belo Horizonte: FIOCRUZ, Centro de Pesquisas René Rachou; 2014. (Série Esquistossomose; 16).

18 Ruiz JM. Índices cercáricos específicos do Schistosoma mansoni verificados em Neves e Mariana, Estado de Minas Gerais. Mem Inst Butantan. 1952;24(1):63-5.

19 Naruto T. Guia para identificação de cercárias. São Paulo: Superintendência de Controle de Endemias do Estado de São Paulo; 1984. 61 p.

20 Frandsen F, Christensen NO. An introductory guide to the identification of cercariae from African freshwater snails with special reference to cercariae of trematode species of medical and veterinary importance. Acta Trop. 1984 Jun;4 1 (2): 181-202.

21 Schell SC. How to know the trematodes. Dubuque (USA): W. C. Brown Company; 1970. 355 p.

22 Boaventura MF, Fernandez MA, Thiengo SC, Silva RE, Melo AL. Formas larvais de Trematoda provenientes de gastrópodes límnicos da microrregião Rio de Janeiro, sudeste do Brasil. Lundiana. 2002;3(1):45-9.

23 Deslandes N. Técnica de dissecção e exame de planorbídeos. Rev Serv Espec Saude Publica (Rio de Janeiro). 1951;4(2):371-82.

24 Pan American Health Organization. A guide for the identification of the snail intermediate hosts of schistosomiasis in the Americas. Washington: Pan American Health Organization; 1968. 122 p. (Scientific publication; no. 168).

25 Paraense WL. Estado atual da sistemática dos planorbídeos brasileiros. Arq Mus Nac (Rio de Janeiro). 1975;55:105-28.

26 Barbosa FS. Aspectos da biologia de Zygocotyle lunata (Trematoda: Zygocotylidae) isolado de Biomphalaria straminea (Mollusca: Planorbidae) oriundas de Iguatama, Minas Gerais, Brasil [dissertação]. Belo Horizonte (MG): Universidade Federal de Minas Gerais, Instituto de Ciências Biológicas; 2011.58 p.

27 Ximenes RF, Gonçalves ICB, Miyahira IC, Pinto HA, Melo AL, Santos SB. Centrocestus formosanus (Trematoda: Heterophyidae) in Melanoides tuberculata (Gastropoda: Thiaridae) from Vila do Abraão, Ilha Grande, Rio de Janeiro, Brazil. Braz J Biol. 2017 Apr-Jun;77(2):318-22.

28 Murillo EAP. Estudo morfológico e molecular de trematódeos transmitidos por Melanoides tuberculata (Mollusca: Thiaridae) em coleções aquáticas do Peru [dissertação]. Belo Horizonte (MG): Universidade Federal de Minas Gerais, Instituto de Ciências Biológicas; 2017. 100 p. 
29 Locke SA, McLaughlin JD, Lapierre AR, Johnson PTJ, Marcogliese DJ. Linking larvae and adults of Apharyngostrigea cornu, Hysteromorpha triloba, and Alaria mustelae (Diplostomoidea: Digenea) using molecular data. J Parasitol. 2011 Oct;97(5):846-51.

30 Silva RE, Melo AL. Caracterização de larvas de trematódeos emergentes de moluscos de água doce coletados na bacia hidrográfica do Lago Soledade, Ouro Branco, Minas Gerais, Brasil. Lundiana. 2013;1 1(1/2):21-33.

31 Moraes J, Silva MPN, Ohlweiler FP, Kawano T. Schistosoma mansoni and other larval trematodes in Biomphalaria tenagophila (Planorbidae) from Guarulhos, São Paulo State, Brazil. Rev Inst Med Trop S. Paulo. 2009 Mar-Apr;51 (2):77-82.

32 Ruiz JM. Contribuição ao estudo das formas larvárias de trematódeos brasileiros. 2. Fauna de Santos, estado de S. Paulo. Mem Inst Butantan. $1952 ; 24(1): 17-36$.
33 Pinto HA, Melo AL. Melanoides tuberculata (Mollusca: Thiaridae) as an intermediate host of Centrocestus formosanus (Trematoda: Heterophyidae) in Brazil. Rev Inst Med Trop S. Paulo. 2010 Jul-Aug;52(4):207-10.

34 Hung NM, Madsen H, Fried B. Global status of fish-borne zoonotic trematodiasis in humans. Acta Parasitol. 2013 Sep;58(3):231-58.

35 Ohlweiler FP, Eduardo JM, Takahashi FY, Crein GA, Luca LR, Oliveira RC. Larvas de trematódeos associadas a moluscos de água doce em municípios da Região Metropolitana de São Paulo, Estado de São Paulo, Brasil. Rev Pan-Amaz Saude. 2013;4(3):37-48.

36 Artigas PT, Perez DM, Baggio D. Censo coprológico no município de Peruibe (litoral sul do estado de São Paulo). Registro de casos autóctones de esquistossomose mansoni. Rev Saude Publica (Sao Paulo). 1969 dez;3(2):141-7. 